\title{
DESIGN CHARTS FOR VIBRATING THREE LAYERED BEAMS WITH VARIOUS BOUNDARY CONDITIONS
}

\author{
S.H. Farghaly ${ }^{*}$, Y.K. Younes ${ }^{*}$ and R.M. Gad El-Rab ${ }^{* *}$
}

\section{ABSTRACT}

The paper presents comprehensive design charts to be used for optimal selections of multilayered beams with elastic faces. Model deflections and bending were computed through the range of the geometrical and shear parameters which cover soft and stiff core materials. The results include the first three eigen frequencies for four combinations of end conditions of interest in bridge, space-craft and machine designs. The computer aided investigation considered the relative merits with respect to the simple homogeneous beam.

\footnotetext{
* Assistant Professors, ** Graduate Student, Mechanical Design Department, Faculty of Engineering and Technology, MATARIA, Helwan University, Cairo, Egypt.
} 


\title{
INTRODUCTION
}

Recently, multilayered beams, plates, shells and similar configurations are being commonly proposed for vibration reduction and control. The employment of such systems is needed particularly the vibration environment is severe and the structural masses are to be light for acoustical pressure damping. Typical applications are aerospace industry, bridges and modern machinery. The undamped sandwich beam consists of two elastic faces which are usually made of thin and stiff materials separated by a homogeneous or honeycombed core. Cantilever configurations were mainly the object of investigations for both damped and undamped cores, Kerwin, [1j, Di-Taranto, [2], Mead and Markus, [3]. Mead and Markus, [4] worked into the problem of free bending vibration of a three layered undamped systems. They reported a comparative analysis on the effects of two different possible boundary conditions at the free-end. These were the riveted and unrestrained free-ends. The natural frequencies of such beams differ according to the type of end conditions. Usually the analyses and computer calculations are based on different assumptions to remove the complexity of the problem, [3]. Rao, [5] derived the complete set of the equations of motion and boundary conditions which govern the vibration of sandwich beams using the energy approach. He colved them for eight boundary conditions seeking the frequency parameters but for the first two eigenmodes. The numerical difficulties involved in programing the exact solution have been successfully overcome by using a developed iterative approach. In addition, he illustrated his formulae by examples for typical problerns, especially the built in-free beam.

The work presented herein is confined to the complete analysis of the modal and anti-nodal bending problem for four combinations of end conditions at the first three eigenmodes. Design charts were casted to provide optimal selections of the different geometrical and shear parameters for the dynamic bending design of undamped sandwich beams.

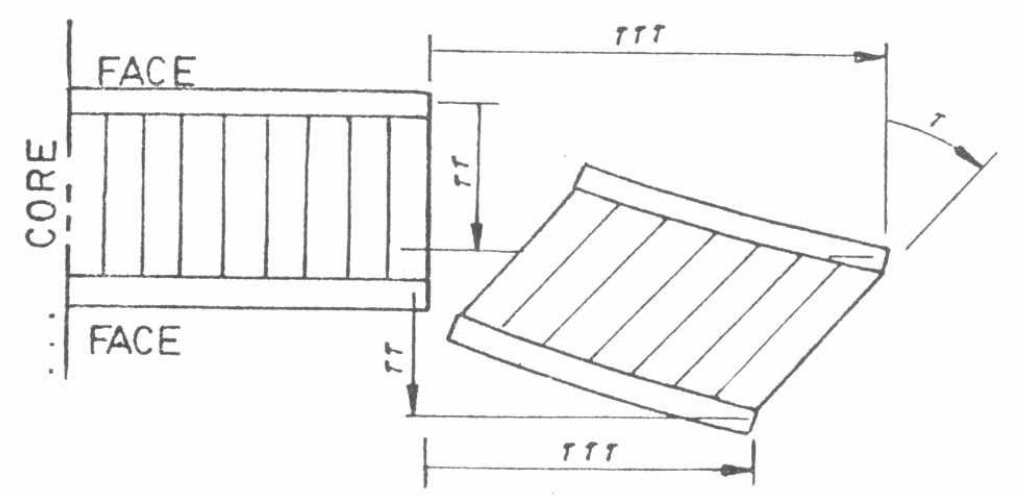

\author{
MEAD SHEAR MODEL \\ - Shear deformation \\ ir Transverse deformation \\ itr Longitudinal deformation
}

According to Nead model, [3] the complex equation of motion for small amoli- 


$$
\begin{aligned}
& \bar{v}_{n}^{V I}-X(1+Y) \bar{v}_{n}^{I V}-a_{n s}^{2}\left[X\left(\eta_{2} \eta_{n}-1\right) \bar{v}_{n}+\bar{v}_{n}^{I I}\right]=0 \\
& \eta_{2} X(1+Y) \bar{v}_{n}-a_{n s}^{2}\left[X\left(\eta_{2}+\eta_{n}\right) \bar{v}_{n}-\eta_{n} \bar{V}_{n}^{I I}\right]=0
\end{aligned}
$$

where the first one is real part and the second is the imaginary part.

For the undamped sandwich beam, the loss factor $\eta_{2}$ which represents the damping in the core is neglected. In this case equation (1-a) reduces to

$$
\bar{V}_{n}^{V I}-X(1+Y) \bar{V}_{n}^{I V}-a_{n s}^{2}\left(\bar{V}_{n}^{I I}-X \bar{V}_{n}\right)=0
$$

where $V_{n}$ is the normal mode of vibration with an expanded solution which may be written in the following form

$$
\begin{aligned}
\bar{v}_{n}= & A_{1} \sin (a \xi)+A_{2} \cos (a \xi)+A_{3} \exp \cdot(b \xi)+A_{4} \exp \cdot(-b \xi) \\
& +A_{5} \exp \cdot(c \xi)+A_{6} \exp \cdot(-c \xi)
\end{aligned}
$$

Six equations can be written according to the beam configuration described by its two end conditions and which are listed in table. 1 .

Table 1. Basic End Conditions

\begin{tabular}{|r|c|c|}
\hline Free end & Clamped end & Pinned end \\
\hline $\bar{v}_{n}^{I I}=0$ & $\bar{v}_{n}=0$ & $\bar{v}_{n}=0$ \\
$\bar{v}_{n}^{I V}-a_{n s}^{2} \bar{v}_{n}=0$ & $\bar{v}_{n}^{I}=0$ & $\bar{v}_{n}^{I I}=0$ \\
\hline
\end{tabular}

In matrix form equations can be written as:

$$
\left[e_{i j}\right]\left[A_{j}\right]=[o]
$$

where $e_{i j}$ are the matrix elements (see appendix A).

For a nontrivial solution and starting with initial estimates for the frequency parameters $a_{n s}$. The Gaussian elimination technique is used to evaluate the normalized coefficients $A_{j}(j=1,2, \ldots .6)$, Rao, $[5,6]$. Upon substitution in equation (3), the mode shape can be evaluated and subsequently, the modal bendings are deduced. For different geometrical and shear parameters (Y, X), anti-nodal values of the dynamic bending moment along the beam span can be specified for best designs concerning dynamic stress values.

\section{DISCUSSION OF RESULTS}

Referring to Figures 1 to 4 complete design information concerning the modal and anti-nodal bending for the four beam configurations are reported. The computational results are presented in graphical format as functions of the geometrical and shear parameters for the first three eigenmodes.

Considering the free-free end conditions, results plotted in Fig. 1, show an increase in the shear parameter $X$ (stiffer core material) leads to an increase in the modal bending, especially for the higher modes. Negligible effects are 
to Figs. 1-b the anti-nodal values show peaks arround $X=1.70$ for the first mode, and for all values of $Y$. The peaks for the second mode are shifted around $X=2.70$ while for the third mode they are around $X=3.70$. These regions represent undesirable design selections, neglecting the very stiff core region. For the clamped-clamped end conditions shown in Figs. 2-b, the antinodal bending for the larger values of $X$ increase as $Y$ increases except for $X \geqslant 50$ at the first mode. The shear parameter $X$ has no effect on the modal bending beyond the value of 100 and $Y=20$ as is clearly shown in Fig. 2-a. For the clamped-free end (Figs. 3-a,b), the anti-nodal peaks are relatively observed for high $X$ values especially for higher modes [7]. For the pinnedpinned end conditions where flexibility exsist to the effect of the shear parameter $X$ is pronounced and the anti-nodal bending values increase gradually with the shear parameter $X$ as shown in Figs. 4-a,b. In all cases, [7] and [8] the . configuration with the geometrical parameter $Y \leqslant 1$ slight changes are observed over the investigated $X$ values for the anti-nodal modal bending values (homogeneous beam cases).

\section{CONCLUSION}

Design charts have been reported for four practical multi-layered undamped beam configurations of the shear model. Specific information concerning the modal and anti-nodal modal bending moment for different geometrical and shear parameters for the first three eigenmodes are provided. The work presented herin is based on an extensive runs of the developed computer program which analyzes sandwich beam configurations and can be used in bridge, spacecraft, and modern machinery designs.

\section{REFERENCES}

1. Kerwin, E.M., "Damping of Flexural Waves by a Constrained Viscoelastic Layer", J. Acoust. Soc. Am. 31, 7. JR (1959).

2. Di-Taranto, R.A., "Theory of Vibratory Elastic and Viscoelastic Layered Finite Length Beams", J. Appl. Mechanics, 32, Trans. ASME Vol. 87, Series E, (1965).

3. Mead, D.J. and Markus, S., "The Forced Vibration of a Three Layered Damped Sandwich Beam with Arbitrary Boundary Conditions", J. Sound Vib. 10 (2), Sept. (1969).

4. Mead, D.J. and Markus, S., "On The Problem of Bending Vibration of Sandwich Cantilever with Various Boundary Conditions Applied at the Free End", STROJNICKY CASOPIS, ROCNIN XXI, CISLO 1, (1970).

5. Rao, D.K., "Frequency and Loss Factors of Sandwich Beams Under Various Boundary Conditions", J. of Mech. Eng. Science, Vol. 20 No 5, (1978).

6. Rao, S.S., "Finite Element Method in Engineering", Pergamon, (1982)

7. Farghaly, S.H., "A Model Analysis of A Vibrating Sandwich Cantilever Beam", ASA T First Conf., VB-2, pp 525-532, May (1985).

8. Gad el-Rab, R.M., "Analytical and Experimental Studies in The Statics and Dynamics of Bonded Multilayered Beams", M.Sc. Thesis Mech. Design Dept.,
Helwan Univ., Oct. (1985). 
\begin{tabular}{|l|l|}
\hline DYN-11 & 119 \\
\hline
\end{tabular}

SECOND A.M.E. CONFERENCE

6 - 8 May 1986 , Cairo

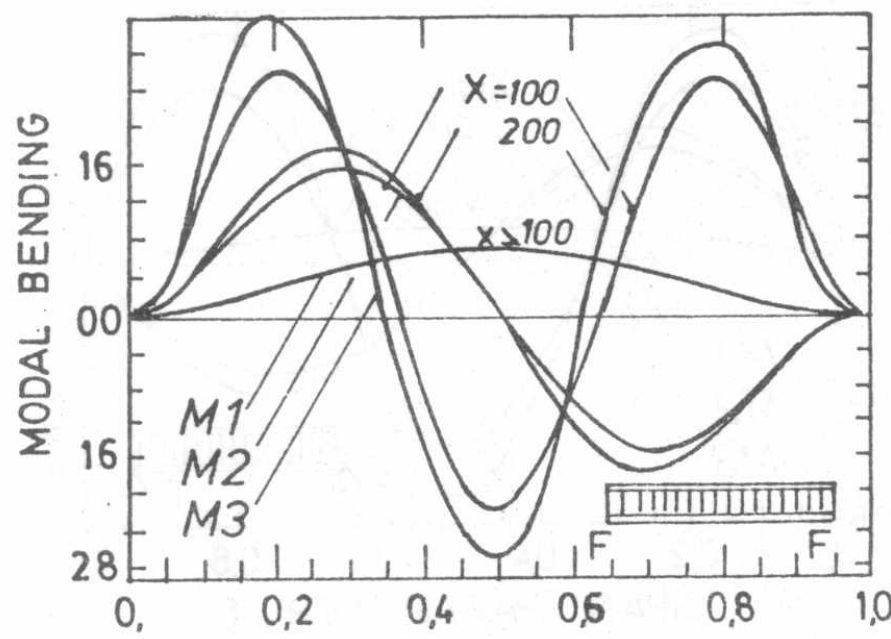

(a)

SPAN PARAMETER, $;$

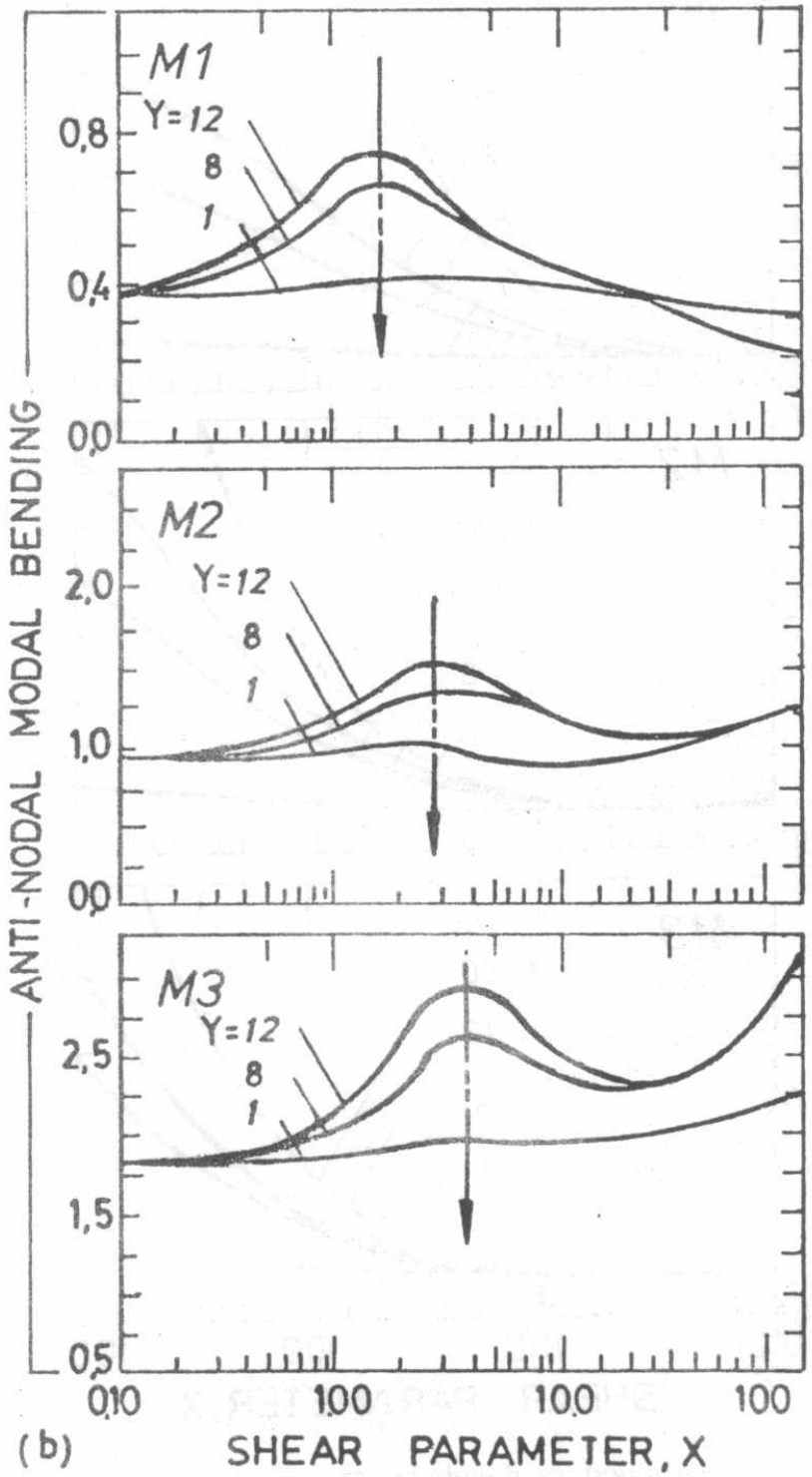

Fig.1: Free-free sandwich beam

a. Modal bending for $Y=20$ and different $v$ alues of $x$.

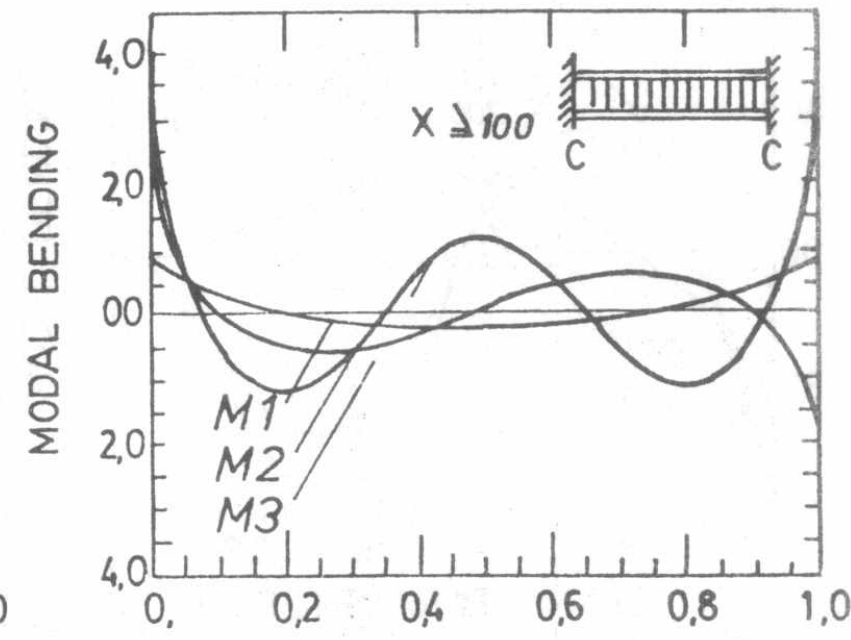

(व)
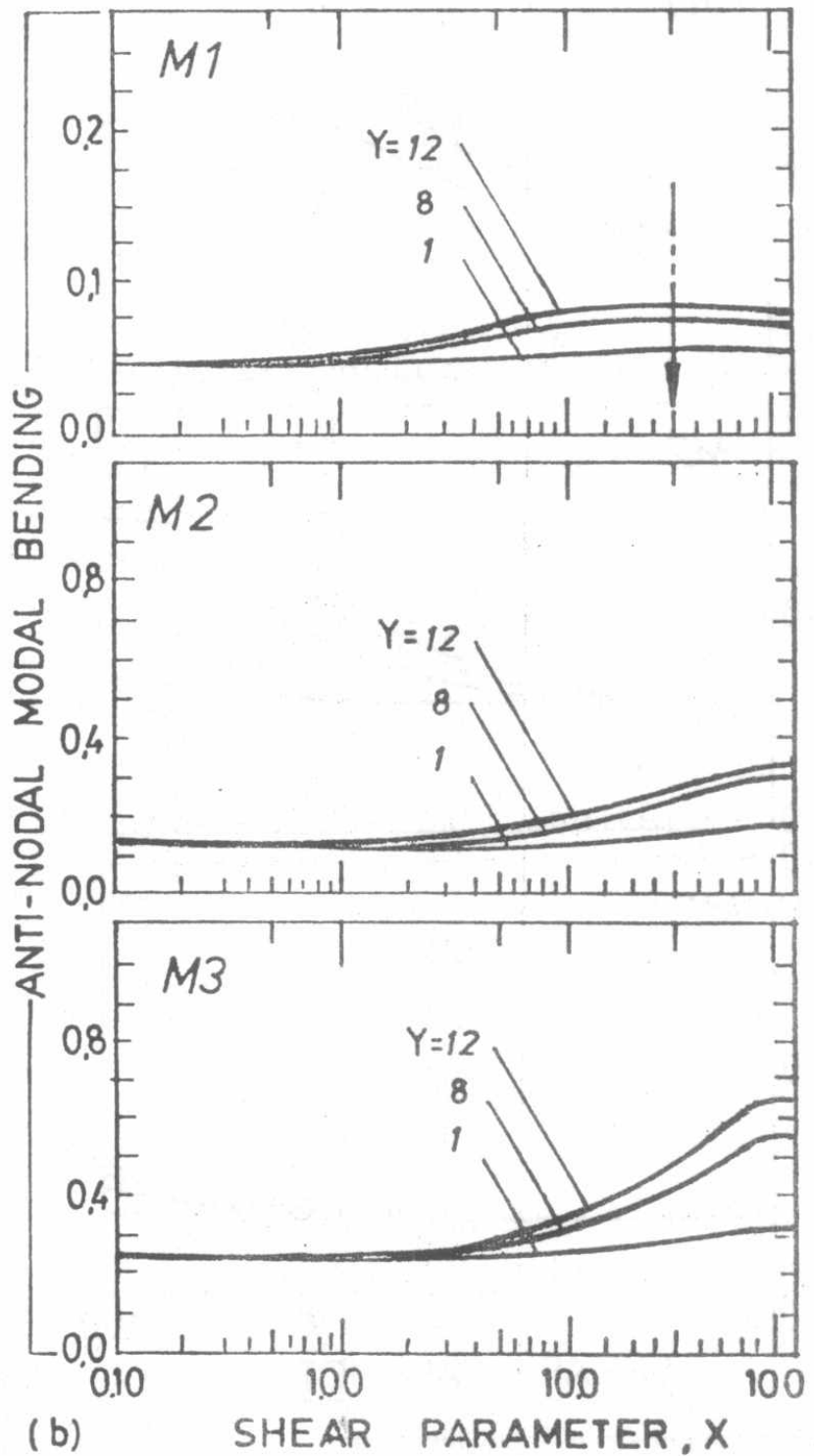

Iig-2: Clamped-clamped sandwich beam

a. Modal bending for $Y=20$ and different $v$ alues of $x$. 
\begin{tabular}{|l|l|}
\hline DYN-1] 120 \\
\hline
\end{tabular}

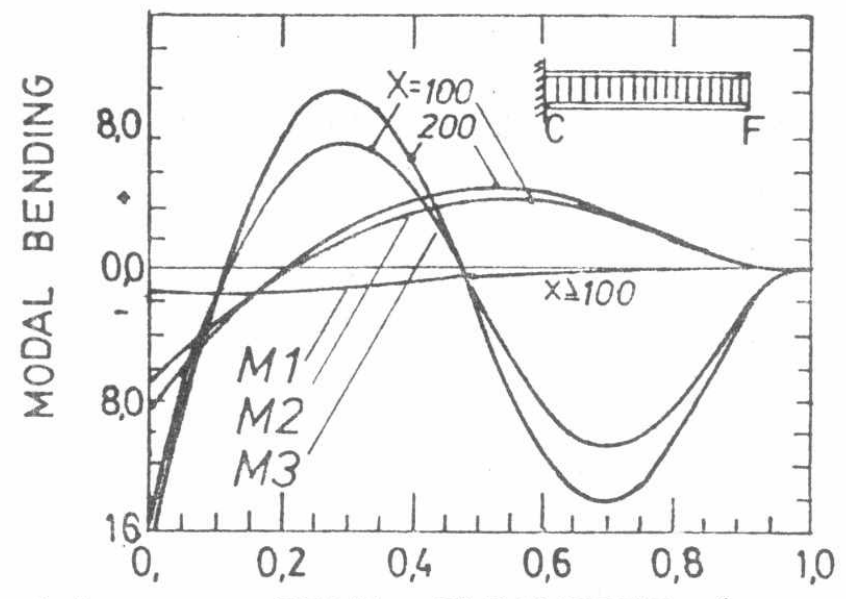

(a)

SPAN PARAMETER, $\xi$

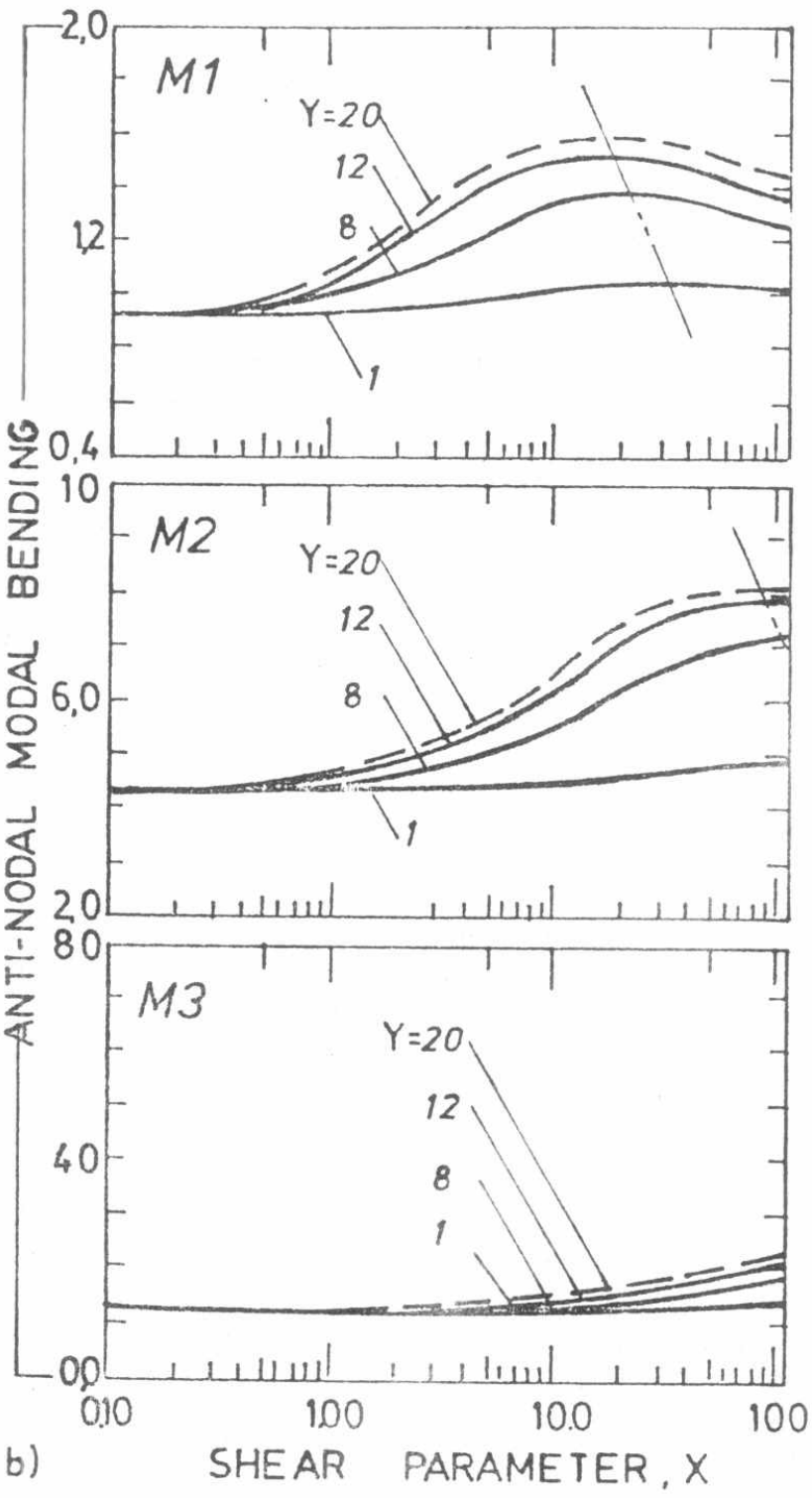

Flg3: Clamped-free sandwich beam
a. Modal bending for $Y=20$ and different values of $x$.

b. Anti-nodal bending for different $X$ and

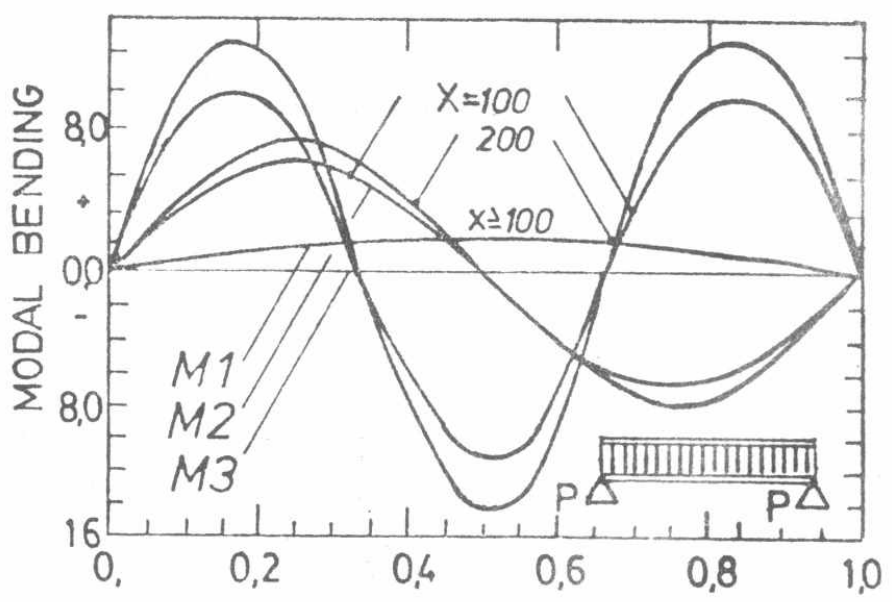

(a)
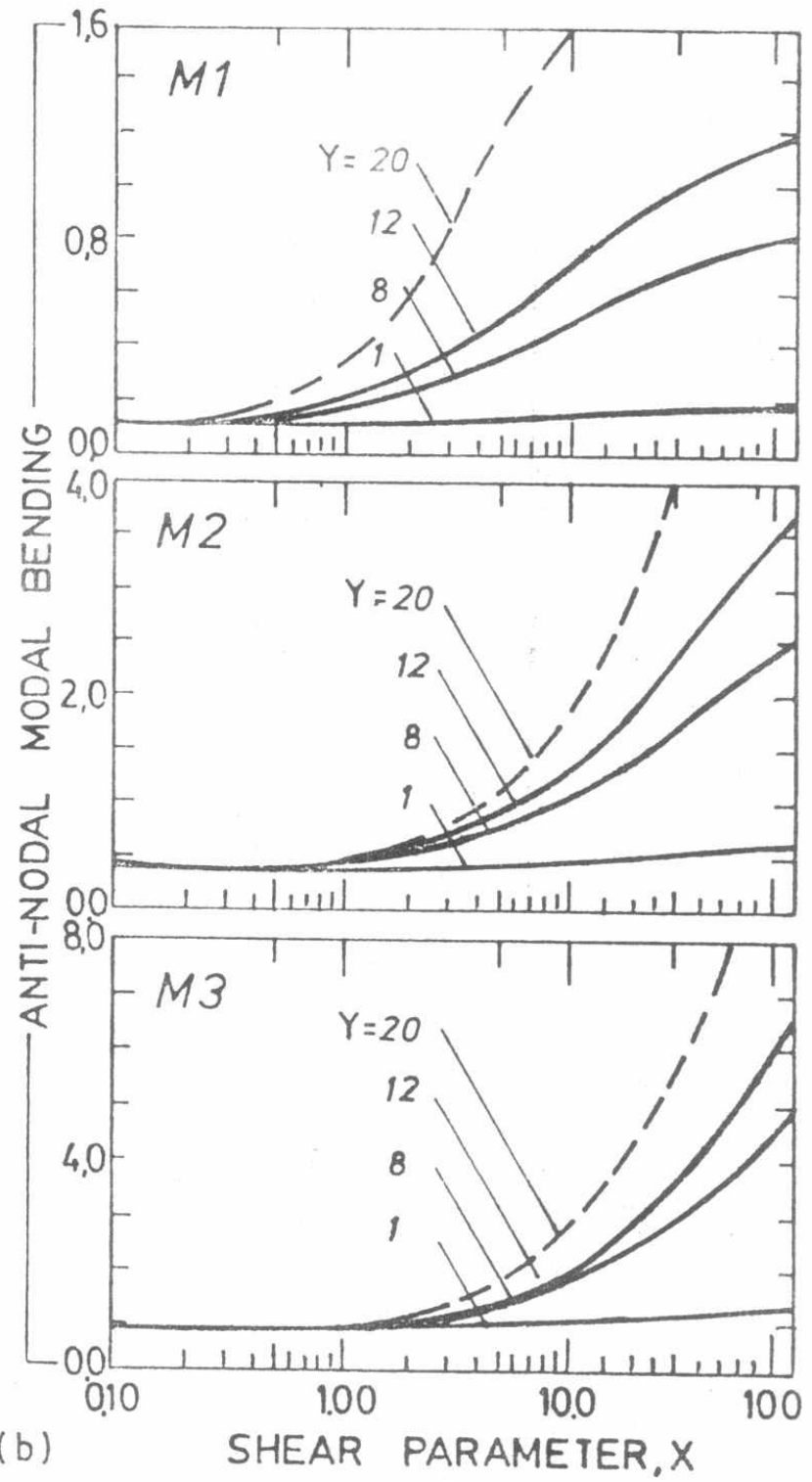

Fig-4: Pinned-Pinned sandwich beam

a. Modal bending for $Y=20$ and different values of $x$.

b. Anti-nodal bernding for different $X$ and 
DYN-11 121

NOMENCLATURE

\section{Latin Letters}

$\begin{array}{ll}\text { a,b,c } & \text { Characteristics equation roots } \\ a_{n s} & \text { Model frequency parameter }=\omega_{n} \sqrt{\mathrm{mL}^{4} / \mathrm{B}} \\ \mathrm{B} & \text { Flexural Rigidity }=(\mathrm{EI})_{\mathrm{i}}, \mathrm{i}=1,3 \\ \mathrm{C} & \text { Central distance between face layers } \\ \mathrm{E}_{\mathrm{i}} & \text { Young's modulus of } \mathrm{ith} \text { layer } \\ \mathrm{G}_{2} & \text { Core material shear modulus } \\ \mathrm{H}_{\mathrm{i}} & \text { Half thickness of ith layer } \\ \mathrm{I}_{\mathrm{i}} & \text { Area moment of iner tia of } \mathrm{ith} \text { layer about its own midline } \\ \mathrm{K}_{\mathrm{i}} & \text { Longitudinal stiffness of face layers, } \mathrm{i}=1,3 \\ \mathrm{~L} & \text { Beam span } \\ \mathrm{m} & \text { Mass of beam per unit length } \\ \mathrm{X} & \text { Shear parameter }=\mathrm{G}_{2} \mathrm{~L}^{2}\left(\mathrm{~K}_{1}+\mathrm{K}_{3}\right) / 2 \mathrm{H}_{2} \mathrm{~K}_{1} \mathrm{~K}_{3} \\ \mathrm{Y} & \text { Geometrical parameter }=\mathrm{c}^{2} \mathrm{~K}_{1} \mathrm{~K}_{3} / \mathrm{B}\left(\mathrm{K}_{1}+\mathrm{K}_{3}\right)\end{array}$

\section{Greek Letters}

$\omega_{\mathrm{n}} \quad$ n th modal frequency

$\eta_{2}$ Loss factor of the core material

$\eta_{n} \quad$ Loss factor of the sandwich beam

\} Length ratio (X/L), (Span parameter)

\section{Superscripts for Letters}

I, .., VI Designates the derivation with respect to, $X$.

Designates the non dimensional values. 


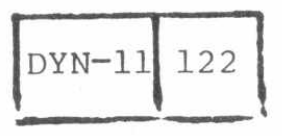

\section{APPENDIX}

Elements of the matrix $\left[\mathrm{e}_{i j}\right]$
Free-free sandwich beam

$$
\begin{aligned}
e_{11} & =0 \\
e_{12} & =-a^{2} \\
e_{13} & =b^{2} \\
e_{14} & =b^{2} \\
e_{15} & =c^{2} \\
e_{16} & =c^{2} \\
e_{21} & =0 \\
e_{22} & =a^{4}-a_{n s}^{2} \\
e_{23} & =b^{4}-a_{n s}^{2} \\
e_{24} & =b^{4}-a_{n s}^{2} \\
e_{25} & =c^{4}-a_{n s}^{2} \\
e_{26} & =c^{4}-a_{n s}^{2} \\
e_{31} & =a^{5}+X(1+Y) a^{3}-a \cdot a_{n s}^{2} \\
e_{32} & =0 \\
e_{33} & =b^{5}-X(1+Y) b^{3}-b \cdot a_{n s}^{2} \\
e_{34} & =-e_{33} \\
e_{35} & =c^{5}-X(1+Y) c^{3}-c \cdot a_{n s}^{2} \\
e_{36} & =-e_{35}
\end{aligned}
$$

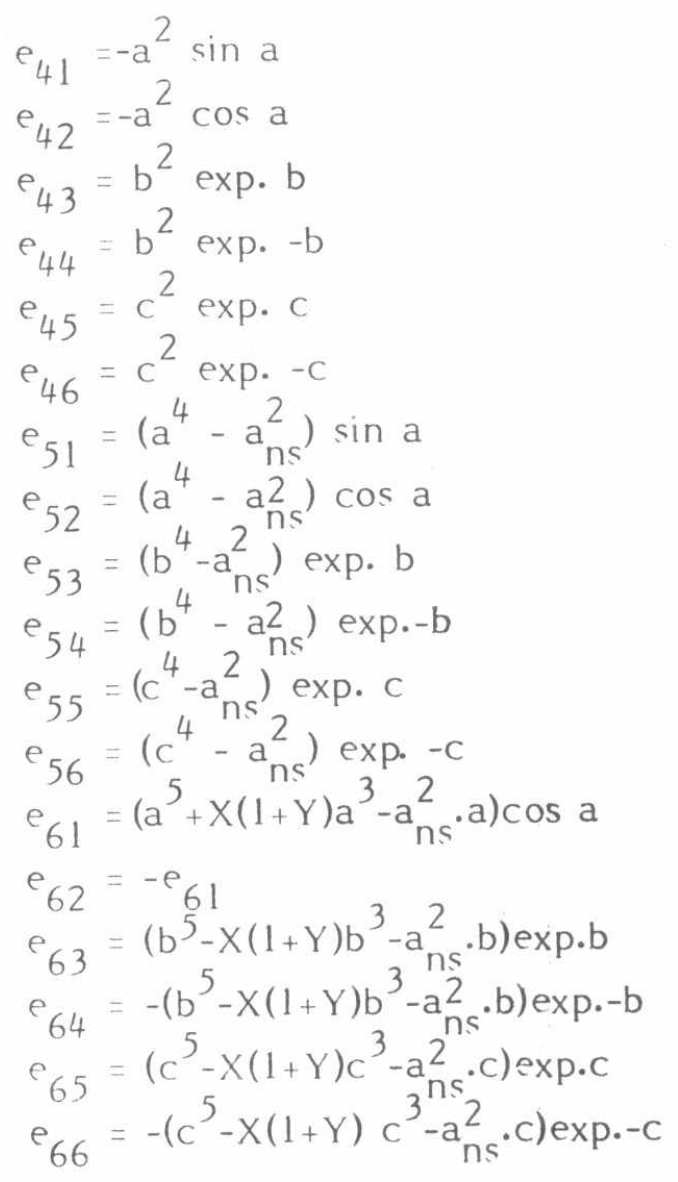

Clamped-clamped sandwich beam

$$
\begin{aligned}
& e_{11}=0 \\
& e_{12}=e_{13}=e_{14}=e_{15}=e_{16}=1 \\
& e_{21}=a \\
& e_{22}=0 \\
& e_{23}=-e_{24}=b \\
& e_{25}=-e_{26}=c \\
& e_{31}=a^{3}\left(a^{2}+X Y\right) \\
& e_{32}=0 \\
& e_{33}=-e_{34}=b^{3}\left(b^{2}-X Y\right) \\
& e_{35}=-e_{36}=c^{3}\left(c^{2}-X Y\right) \\
& e_{41}=\sin a \\
& e_{42}=\cos a \\
& e_{43}=\exp \cdot b
\end{aligned}
$$

$$
\begin{aligned}
& e_{45}=\exp \cdot c \\
& e_{46}=\exp \cdot-c \\
& e_{51}=a \cos a \\
& e_{52}=-a \sin a \\
& e_{53}=b \exp \cdot b \\
& e_{54}=-b \exp \cdot-b \\
& e_{55}=c \exp \cdot c \\
& e_{56}=-c \exp \cdot-c \\
& e_{61}=\left(a^{5}+X Y a^{3}\right) \cos a \\
& e_{62}=-\left(a^{5}+X Y a^{3}\right) \sin a \\
& e_{63}=\left(b^{5}-X Y b^{3}\right) \text { exp. b } \\
& e_{64}=-\left(b^{5}-X Y b^{3}\right) \text { exp. -b } \\
& e_{65}=\left(c^{5}-X Y c^{3}\right) \text { exp. } c
\end{aligned}
$$


Clamped-free sandwich beam

$$
\begin{aligned}
& e_{11}=o \\
& e_{12}=e_{13}=e_{14}=e_{15}=e_{16}=1 \\
& e_{21}=a \\
& e_{22}=0 \\
& e_{23}=-e_{24}=b \\
& e_{25}=-e_{26}=c \\
& e_{31}=a^{3}\left(a^{2}+X Y\right) \\
& e_{32}=0 \\
& e_{33}=-e_{34}=b^{3}\left(b^{2}-X Y\right) \\
& e_{35}=-e_{36}=c^{3}\left(c^{2}-X Y\right) \\
& e_{41}=-a^{2} \sin a \\
& e_{42}=-a^{2} \cos a \\
& e_{43}=b^{2} \exp \cdot b \\
& e_{44}=b^{2} \exp \cdot-b
\end{aligned}
$$

$e_{45}=c^{2}$ exp. $c$

$e_{46}=c^{2}$ exp. $-c$

$e_{51}=\left(a^{4}-a_{n s}^{2}\right) \sin a$

$e_{52}=\left(a^{4}-a_{n s}^{2}\right) \cos a$

$e_{53}=\left(b^{4}-a_{n s}^{2}\right)$ exp. b

$e_{54}=\left(b^{4}-a_{n s}^{2}\right)$ exp. - b

$e_{55}=\left(c^{4}-a_{n s}^{2}\right)$ exp. $c$

$e_{56}=\left(c^{4}-a_{n s}^{2}\right)$ exp. $-c$

$e_{61}=\left[a^{4}+a^{2} X(1+Y)-a_{n s}^{2}\right] a \cos a$

$e_{62}=\left[-a^{4}-a^{2} X(1+Y)+a_{n s}^{2}\right] a \sin a$ $e_{63}=\left[b^{4}-b^{2} X(1+Y)-a_{n s}^{2}\right) b \cdot$ exp. b $e_{64}=\left[-b^{4}+b^{2} X(1+Y)+a_{n s}^{2}\right]$ b. exp. -b $e_{65}=\left[c^{4}-c^{2} X(1+Y)-a_{n s}^{2}\right] c \cdot$ exp. $c$ $e_{66}=\left[-c^{4}+c^{2} X(1+Y)+a_{n s}^{2}\right] c \cdot$ exp. $-c$

pinned-pinned sandwich beam

$$
\begin{aligned}
& e_{11}=0 \\
& e_{12}=e_{13}=e_{14}=e_{15}=e_{16}=0 \\
& e_{21}=0 \\
& e_{22}=-a^{2} \\
& e_{23}=e_{24}=b^{2} \\
& e_{25}=e_{26}=c^{2} \\
& e_{31}=0 \\
& e_{32}=a^{4} \\
& e_{33}=e_{34}=b^{4} \\
& e_{35}=e_{36}=c^{4} \\
& e_{41}=\sin a \\
& e_{42}=\cos a \\
& e_{43}=\operatorname{exp.} b
\end{aligned}
$$

$$
\begin{aligned}
& e_{44}=\exp \cdot-b \\
& e_{45}=\exp \cdot c \\
& e_{46}=\exp \cdot-c \\
& e_{51}=a^{2} \sin a \\
& e_{52}=-a^{2} \cos a \\
& e_{53}=b^{2} \cdot \exp \cdot b \\
& e_{54}=b^{2} \exp \cdot-b \\
& e_{55}=c^{2} \exp \cdot c \\
& e_{56}=c^{2} \exp \cdot-c \\
& e_{61}=a^{4} \sin a \\
& e_{62}=a^{4} \cos a \\
& e_{63}=b^{4} \exp \cdot b \\
& e_{64}=b^{4} \exp \cdot-b \\
& e_{65}=c^{4} \exp \cdot c \\
& e_{66}=c^{4} \exp \cdot-c
\end{aligned}
$$


